\title{
The Existence and Simulations of Periodic Solution of Predator-prey Models with Impulsive Perturbations and Holling Type III Functional Responses
}

\author{
Kaihua Wang \\ School of Mathematics and Statistics \\ Hainan Normal University \\ Haikou, Hainan, 571158, P.R. China
}

\author{
Zhanji Gui* \\ Department of Software Engineering \\ Hainan College of Software Technology \\ Qionghai, 571400, P.R. China \\ *Corresponding author
}

\begin{abstract}
In this paper, a criterion for the existence of periodic solutions of predator-prey models with impulsive perturbations and Holling type III functional responses is established using the continuation theorem of coincidence degree theory and analysis techniques. Further, some numerical simulations show that our models can occur in many forms of complexities including periodic oscillation and Gui strange attractors.
\end{abstract}

Keywords-periodic solution; predator-prey model; coincidence degree theory; impulses

\section{INTRODUCTION}

The prey-predator systems of Lotka-Volterra type have been discussed widely in the last century. In the literature many studies considered the predator species as density independence. However, considerable evidence show that some predator species may be density dependence because of the environmental factors $[1,2,3]$. It is more appropriate to add the density-dependent term to these models in such a circumstance. On the other hand, many systems arising in physical, chemical and biological phenomena exhibit impulsive dynamical behaviours due to the abrupt jumps during the evolution processes, which can be modelled by impulsive differential equations [4-9]. In this paper, we shall explore the dynamics of the nonautonomous predator-prey system with impulsive perturbations and Holling type III functional responses in a more general form.

$$
\left\{\begin{array}{l}
\frac{d y_{1}(t)}{d t}=y_{1}(t)\left[r(t)-a(t) y_{1}(t)\right]-\frac{b(t) y_{1}^{2}(t) y_{2}(t)}{\beta^{2}(t)+y_{1}^{2}(t)}, \\
\frac{d y_{2}(t)}{d t}=-d(t) y_{2}(t)+\frac{c(t) y_{1}^{2}(t) y_{2}(t)}{\beta^{2}(t)+y_{1}^{2}(t)}-e(t) y_{2}^{2}(t), \\
\Delta y_{1}\left(t_{k}\right)=c_{k} y_{1}\left(t_{k}\right), \\
\Delta y_{2}\left(t_{k}\right)=d_{k} y_{2}\left(t_{k}\right),
\end{array}\right\} t=t_{k}, k=1,2, \ldots
$$

where $y_{1}(t)$ and $y_{2}(t)$ represent densities of prey and predator at time $t$ respectively. $r(t), a(t), b(t), c(t)$, $d(t), e(t), \beta(t)$ are positive periodic continuous functions with period $\omega \quad, \quad 0<t_{1}<t_{2}<\cdots<t_{k}<\cdots \quad$ and $\lim _{t \rightarrow+\infty} t_{k}=+\infty$. Assume that $c_{k}, d_{k}\left(k \in \mathbb{Z}_{+}\right)$are constants and there exists a positive integer $q$ such that $t_{k+q}=t_{k}+\omega$, $c_{k+q}=c_{k}, d_{k+q}=d_{k}$ and $0<t_{k+1}-t_{k}<\omega$.

Let $g(t)$ be a bounded continuous function on $\mathbb{R}$. Define $g^{l}=\inf _{t \in R} g(t), g^{u}=\sup _{t \in R} g(t)$. Particularly, if $g(t)$ are $\omega$ periodic function with respect to $t$, then

$$
\bar{g}=\frac{1}{\omega} \int_{0}^{\omega} g(t) d t .
$$

\section{EXISTENCE OF POSITIVE PERIODIC SOLUTIONS}

To prove our results, we need the notion of the Mawhin's continuation theorem formulated in [10].

Lemma 1. Let $X$ and $Y$ be two Bannach space. Consider an operator equation $L x=\lambda N x$, where $L: \operatorname{Dom} L \cap X \rightarrow Y$ is a Fredholm operator of index zero and $\lambda \in[0,1]$ is a parameter. Then there exist two projectors $P: X \rightarrow \operatorname{Ker} L$ and $Q: Y \rightarrow Y / \operatorname{Im} L$. Assume that $N: \bar{\Omega} \rightarrow Y$ is $L$-compact on $\bar{\Omega}$, where $\Omega$ is open bounded in $X$. Furthermore, assume that:

(a) for each $\lambda \in(0,1), x \in \partial \Omega \cap \operatorname{Dom} L, L x \neq \lambda N x$.

(b) for each $x \in \partial \Omega \cap \operatorname{Ker} L, Q N x \neq 0$,

(c) $\operatorname{deg}\{J Q N, \Omega \cap \operatorname{Ker} L, 0\} \neq 0$, where $J: \operatorname{Im} Q \rightarrow \operatorname{Ker} L$ is an isomorphism and $\operatorname{deg}\{*\}$ represents the Brouwer degree.

Then equation $L x=N x$ has a solution on $\bar{\Omega} \cap \operatorname{Dom} L$.

Now we are ready to state and prove the main results of the present paper.

Theorem 1 Assume that the following conditions hold:

$$
\begin{aligned}
& C_{ \pm}:=2 \bar{r} \omega \pm \ln \prod_{k=1}^{q}\left(1+c_{k}\right)>0, \\
& D_{ \pm}:=2 \bar{d} \omega \pm \ln \prod_{k=1}^{q}\left(1+d_{k}\right)>0,
\end{aligned}
$$




$$
\begin{gathered}
m_{1}:=\frac{1}{2} \ln \frac{\beta^{2 l}\left(D_{-}-\bar{d} \omega\right)}{\bar{c} \omega}-C_{+}>0, \\
M_{1}:=\ln \frac{C_{+}-\bar{r} \omega}{\bar{a} \omega}+C_{+}>0, \\
m_{2}:=\ln \frac{1}{\bar{e}}\left(\frac{c^{l} \omega e^{2 m_{1}}}{\omega^{2 u}+e^{2 m_{1}}}-D_{-}+\bar{d} \omega\right)-D_{+}>0, \\
M_{2}:=\ln \frac{\bar{c} \omega-D_{-}}{\bar{e} \omega}+D_{+}>0
\end{gathered}
$$

then system (1) has at least one $\omega$-periodic solution.

Proof. Make the change of variables $y_{1}(t)=\exp \left\{x_{1}(t)\right\}$, $y_{2}(t)=\exp \left\{x_{2}(t)\right\}$, then system (1) can be reformulated as

$$
\left\{\begin{array}{c}
\frac{d x_{1}(t)}{d t}=r(t)-a(t) e^{x_{1}(t)}-\frac{b(t) e^{x_{1}(t)+x_{2}(t)}}{\beta^{2}(t)+e^{2 x_{1}(t)}}, \\
\frac{d x_{2}(t)}{d t}=-d(t)+\frac{c(t) e^{2 x_{1}(t)}}{\beta^{2}(t)+e^{2 x_{1}(t)}}-e(t) e^{x_{2}(t)} \\
\Delta x_{1}\left(t_{k}\right)=\ln \left(1+c_{k}\right), \\
\Delta x_{2}\left(t_{k}\right)=\ln \left(1+d_{k}\right),
\end{array}\right\} t=t_{k}, k=1,2, \ldots
$$

Let

$$
P C(J, \mathbb{R})=\left\{\begin{array}{l}
x: J \rightarrow \mathbb{R} \mid x(t) \text { is continuous with respect to } \\
t \neq t_{1}, \cdots, t_{q} ; x\left(t^{+}\right) \text {and } x\left(t^{-}\right) \text {exist at } t_{1}, \cdots, t_{q} ; \\
\text { and } x\left(t_{k}\right)=x\left(t_{k}^{-}\right), k=1,2, \cdots, q
\end{array}\right\}
$$

To complete the proof, we only need to search for an appropriate open bounded subset $\Omega \subset X$ verifying all the requirements in Lemma 1.

Note $x=\left(x_{1}, x_{2}\right)^{T}$, define

$$
X=\left\{x \in P C\left(\mathbb{R}, \mathbb{R}^{2}\right): x(t+\omega)=x(t)\right\},
$$

$Y=X \times \mathbb{R}^{2 q}$, then it is standard to show that both $X$ and $Y$ are Banach space when they are endowed with the norms $\|x\|_{c}=\sup _{t \in[0, \omega]}|x(t)|$ and

$$
\left\|\left(x, c_{1}, \cdots, c_{q}\right)\right\|=\left(\|x\|_{c}^{2}+\left|c_{1}\right|^{2}+\cdots+\left|c_{q}\right|^{2}\right)^{1 / 2} .
$$

Let

$$
\operatorname{Dom} L=\left\{x \in C^{1}\left[0, \omega ; t_{1}, \cdots, t_{q}\right] \mid x(0)=x(\omega)\right\},
$$

$L: \operatorname{Dom} L \rightarrow Y, L x=\left(x^{\prime}, \Delta x\left(t_{1}\right), \cdots, \Delta x\left(t_{q}\right)\right), N: X \rightarrow Y$,

$$
\begin{aligned}
N x & =N\left(\begin{array}{l}
x_{1}(t) \\
x_{2}(t)
\end{array}\right) \\
& \left.\left.=\left(\begin{array}{c}
r(t)-a(t) e^{x_{1}(t)}-\frac{b(t) e^{x_{1}(t)+x_{2}(t)}}{\beta^{2}(t)+e^{2 x_{1}(t)}} \\
-d(t)+\frac{c(t) e^{2 x_{1}(t)}}{\beta^{2}(t)+e^{2 x_{1}(t)}}-e(t) e^{x_{2}(t)}
\end{array}\right),\left(\begin{array}{l}
\ln \left(1+c_{k}\right) \\
\ln \left(1+d_{k}\right)
\end{array}\right)\right)_{k=1}^{q}\right) .
\end{aligned}
$$

It is easy to prove that $L$ is a Fredholm operator of index zero. Consider the operator equation

$$
L x=\lambda N x, \lambda \in(0,1) .
$$

Integratinrg (3) over the interval $[0, \omega]$, we obtain

$$
\left\{\begin{array}{r}
\int_{0}^{\omega}\left[r(t)-a(t) e^{x_{1}(t)}-\frac{b(t) e^{x_{1}(t)+x_{2}(t)}}{\beta^{2}(t)+e^{2 x_{1}(t)}}\right] d t \\
=-\sum_{k=1}^{q} \ln \left(1+c_{k}\right)=\ln \prod_{k=1}^{q} \frac{1}{1+c_{k}}, \\
\int_{0}^{\omega}\left[-d(t)+\frac{c(t) e^{2 x_{1}(t)}}{\beta^{2}(t)+e^{2 x_{1}(t)}}-e(t) e^{x_{2}(t)}\right] d t \\
=-\sum_{k=1}^{q} \ln \left(1+d_{k}\right)=\ln \prod_{k=1}^{q} \frac{1}{1+d_{k}} .
\end{array}\right.
$$

It follows that

$$
\begin{gathered}
\bar{r} \omega-\ln \prod_{k=1}^{q} \frac{1}{1+c_{k}}=\int_{0}^{\omega} a(t) e^{x_{1}(t)} d t+\int_{0}^{\omega} \frac{b(t) e^{x_{1}(t)+x_{2}(t)}}{\beta^{2}(t)+e^{2 x_{1}(t)}} d t \\
\bar{d} \omega+\ln \prod_{k=1}^{q} \frac{1}{1+d_{k}}=\int_{0}^{\omega} \frac{c(t) e^{2 x_{1}(t)}}{\beta^{2}(t)+e^{2 x_{1}(t)}}-\int_{0}^{\omega} e(t) e^{x_{2}(t)} d t .
\end{gathered}
$$

We can derive

$$
\begin{array}{r}
\int_{0}^{\omega}\left|x_{1}^{\prime}(t)\right| d t \leq 2 \bar{r} \omega+\ln \prod_{k=1}^{q}\left(1+c_{k}\right)=C_{+}, \\
\int_{0}^{\omega}\left|x_{2}^{\prime}(t)\right| d t \leq 2 \bar{d} \omega+\ln \prod_{k=1}^{q}\left(1+d_{k}\right)=D_{+} .
\end{array}
$$

Since $\quad x_{i}(t) \in P C([0, \omega], \mathbb{R}), \quad$ there exist $\xi_{i}, \eta_{i} \in[0, \omega] \cup\left\{t_{1}^{+}, t_{2}^{+}, \ldots, t_{p}^{+}\right\}$, such that

$$
x_{i}\left(\xi_{i}\right)=\inf _{0 \leq t \leq \omega}\left|x_{i}(t)\right|, \quad x_{i}\left(\eta_{i}\right)=\sup _{0 \leq t \leq \omega}\left|x_{i}(t)\right|, i=1,2 .
$$

From (4) and (5), we can see 


$$
\begin{aligned}
& \int_{0}^{\omega} a(t) e^{x_{1}\left(\xi_{1}\right)} d t \leq \int_{0}^{\omega} a(t) e^{x_{1}(t)} d t \\
& \leq \bar{r} \omega-\ln \prod_{k=1}^{q} \frac{1}{1+c_{k}} \\
&=\bar{r} \omega+\ln \prod_{k=1}^{q}\left(1+c_{k}\right), \\
& \int_{0}^{\omega} e(t) e^{x_{2}\left(\xi_{2}\right)} d t \leq \int_{0}^{\omega} e(t) e^{x_{2}(t)} d t \\
& \leq \int_{0}^{\omega} c(t) d t-\bar{d} \omega-\ln \prod_{k=1}^{q} \frac{1}{1+d_{k}} \\
&=(\bar{c}-\bar{d}) \omega+\ln \prod_{k=1}^{q}\left(1+d_{k}\right),
\end{aligned}
$$

It follows that

$$
\begin{gathered}
x_{1}\left(\xi_{1}\right) \leq \ln \frac{\bar{r} \omega+\ln \prod_{k=1}^{q}\left(1+c_{k}\right)}{\bar{a} \omega} \\
x_{2}\left(\xi_{2}\right) \leq \ln \frac{(\bar{c}-\bar{d}) \omega+\ln \prod_{k=1}^{q}\left(1+d_{k}\right)}{\bar{e} \omega}
\end{gathered}
$$

On the other hand, by (5), we also have

$$
\begin{aligned}
\bar{d} \omega+\ln \prod_{k=1}^{q} \frac{1}{1+d_{k}} & \leq \int_{0}^{\omega} \frac{c(t) e^{2 x_{1}(t)}}{\beta^{2}(t)+e^{2 x_{1}(t)}} d t \\
& \leq \int_{0}^{\omega} \frac{c(t) e^{2 x_{1}(t)}}{\beta^{2 l}} d t \\
& \leq \frac{\bar{c} \omega}{\beta^{2 l}} e^{2 x_{1}\left(\eta_{1}\right)}
\end{aligned}
$$

It follows that

$$
x_{1}\left(\eta_{1}\right) \geq \frac{1}{2} \ln \frac{\beta^{2 l}}{\bar{c} \omega}\left(\bar{d} \omega-\ln \prod_{k=1}^{q}\left(1+d_{k}\right)\right) .
$$

Then for $\forall t \in[0, \omega]$, by (6), (8) and (10), we have

$$
\begin{aligned}
& x_{1}(t) \leq x_{1}\left(\xi_{1}\right)+\int_{0}^{\omega}\left|x_{1}^{\prime}(t)\right| d t \\
& \leq \ln \frac{\bar{r} \omega+\ln \prod_{k=1}^{q}\left(1+c_{k}\right)}{\bar{a} \omega}+2 \bar{r} \omega+\ln \prod_{k=1}^{q}\left(1+c_{k}\right) \\
&:=M_{1}, \\
& x_{1}(t) \geq x_{1}\left(\eta_{1}\right)-\int_{0}^{\omega}\left|x_{1}^{\prime}(t)\right| d t \\
& \geq \frac{1}{2} \ln \frac{\beta^{2 l}}{\bar{c} \omega}\left(\bar{d} \omega-\ln \prod_{k=1}^{q}\left(1+d_{k}\right)\right)-2 \bar{r} \omega-\ln \prod_{k=1}^{q}\left(1+c_{k}\right) \\
&:=m_{1},
\end{aligned}
$$

then we can derive

$$
\left|x_{1}(t)\right| \leq \max \left\{m_{1}, M_{1}\right\}:=A_{1} .
$$

From (5), we also have

$$
\begin{aligned}
\bar{e} e^{x_{2}\left(\eta_{2}\right)} & \geq \int_{0}^{\omega} e(t) e^{x_{2}(t)} d t \\
& =\int_{0}^{\omega} \frac{c(t) e^{2 x_{1}(t)}}{\beta^{2}(t)+e^{2 x_{1}(t)}} d t-\bar{d} \omega+\ln \prod_{k=1}^{q}\left(1+d_{k}\right) \\
& \geq \frac{c^{l} e^{2 m_{1}}}{\beta^{2 u}+e^{2 m_{1}}} \omega+\ln \prod_{k=1}^{q}\left(1+d_{k}\right)-\bar{d} \omega .
\end{aligned}
$$

It follows that

$$
\begin{aligned}
x_{2}\left(\eta_{2}\right) & \geq \ln \frac{1}{\bar{e}}\left(\frac{c^{l} e^{2 m_{1}}}{\beta^{2 u}+e^{2 m_{1}}} \omega+\ln \prod_{k=1}^{q}\left(1+d_{k}\right)-\bar{d} \omega\right) \\
& :=m_{2} .
\end{aligned}
$$

So, for $\forall t \in[0, \omega]$, we have

$$
\begin{aligned}
x_{2}(t) \geq & x_{2}\left(\eta_{2}\right)-\int_{0}^{\omega}\left|x_{2}^{\prime}(t)\right| d t \\
\geq & \ln \frac{1}{\bar{e}}\left(\frac{c^{l} e^{2 m_{1}}}{\beta^{2 u}+e^{2 m_{1}}} \omega+\ln \prod_{k=1}^{q}\left(1+d_{k}\right)-\bar{d} \omega\right) \\
& \quad-2 \bar{d} \omega-\ln \prod_{k=1}^{q}\left(1+d_{k}\right) \\
:=m_{2} & \\
x_{2}(t) \leq x_{2}\left(\xi_{2}\right)+\int_{0}^{\omega}\left|x_{2}^{\prime}(t)\right| d t & (\bar{c}-\bar{d}) \omega+\ln \prod_{k=1}^{q}\left(1+d_{k}\right) \\
\leq & \ln \frac{\bar{e} \omega}{i=} M_{2},
\end{aligned}
$$

then we can derive

$$
\left|x_{2}(t)\right| \leq \max \left\{m_{2}, M_{2}\right\}:=A_{2} .
$$

Obviously, $A_{1}, A_{2}$ are independent of $\lambda$. Choosing $A>A_{1}+A_{2}, \Omega=\left\{x \in X:\|x\|_{c}<r\right\}$, then $N$ is $L$ compact on $\bar{\Omega}$. So, for $\forall x=\left(\bar{x}_{1}, \bar{x}_{2}\right)^{T} \in \partial \Omega \cap \operatorname{Ker} L$, we have $Q N x \neq 0 \quad$. Let $J: \operatorname{Im} Q \rightarrow X, \quad(d, 0, \ldots, 0) \rightarrow d$. When $x \in \Omega \cap \operatorname{Ker} L$, in view of the assumptions in Mawhin's continuation theorem [10], one obtains, $\operatorname{deg}\{J Q N, \Omega \cap \operatorname{Ker} L, 0\} \neq 0$. By now we have proved that $\Omega$ satisfies all the requirements in Mawhin's continuation theorem (Lemma 1). Hence, (2) has at least one $\omega$-periodic solution $x^{*}(t)=\left(x_{1}^{*}(t), x_{2}^{*}(t)\right)^{T} \quad$ in $\quad \operatorname{Dom} L \cap \bar{\Omega} \quad$. Set $y_{1}^{*}(t)=\exp \left\{x_{1}^{*}(t)\right\} \quad, \quad y_{2}^{*}(t)=\exp \left\{x_{2}^{*}(t)\right\}, \quad$ then $\quad y^{*}(t)=$ $\left(y_{1}^{*}(t), y_{2}^{*}(t)\right)^{T}$ is an $\omega$-periodic solution of (1). The proof is complete. 


\section{AN ILLUSTRATIVE EXAMPLE}

In system (1), we take

$$
\begin{aligned}
& r(t)=2-0.5 \sin t, a(t)=0.2-0.1 \cos t, \\
& b(t)=0.8+0.1 \sin t, c(t)=2+0.4 \sin t, \\
& d(t)=1-0.2 \cos t, e(t)=0.7+0.1 \cos t, \\
& \beta(t)=2-\sin t .
\end{aligned}
$$

If $c_{k}=0.3, d_{k}=1.1, T=\pi / 2, q=4$, then all conditions of Theorem 1 are satisfied, system (1) has a unique $2 \pi$ periodic solution with four pulses (see Fig.1-Fig.3, we take $\left.\left(y_{1}(0), y_{2}(0)\right)^{T}=(1,1)^{T}\right)$. We find the occurrence of sudden changes in the figures of the time-series and phase portrait. The influence of pulse is obvious.

If $T=2$, then $c_{k+q}=c_{k}, d_{k+q}=d_{k}$ is not satisfied. Periodic oscillation of system (1) will be destroyed by impulsive effect. Numeric results show that system (1) has Gui chaotic strange attractor (see Fig. 4) [4-7]. In Fig. 4, we take $\left(y_{1}(0), y_{2}(0)\right)^{T}=(1,1)^{T}$. Every solution of system (1) will finally tend to the Gui chaotic strange attractor.

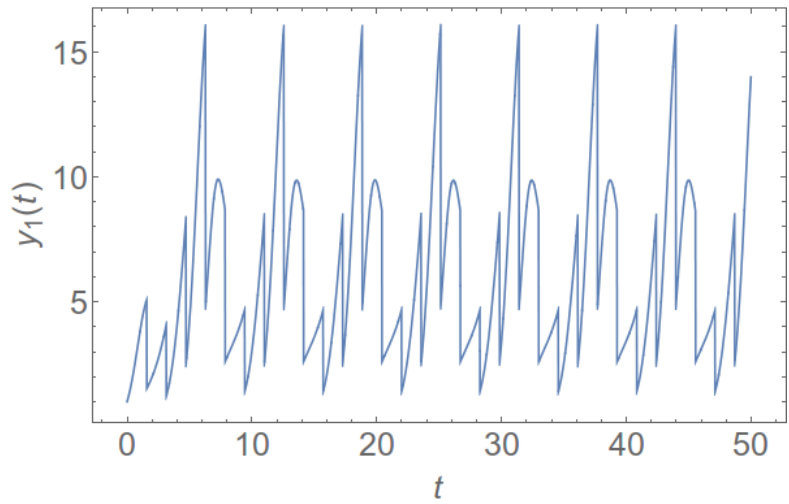

FIGURE I. TIME-SERIES OF $y_{1}(t)$ EVOLVED IN SYSTEM (1)

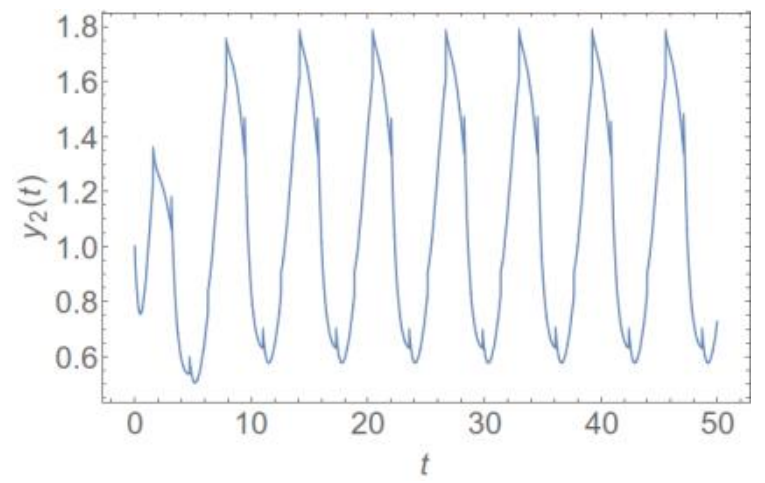

FIGURE II. TIME-SERIES OF $y_{2}(t)$ EVOLVED IN SYSTEM (1)

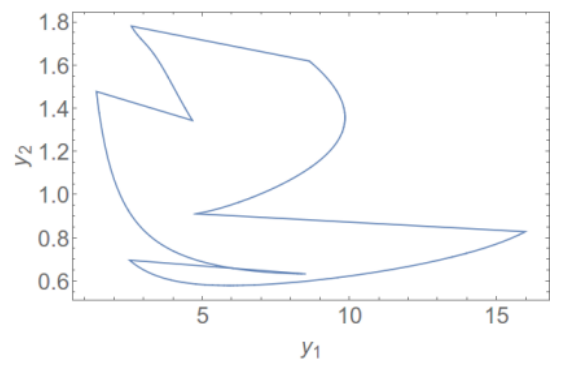

FIGURE III. PHASE PORTRAIT OF PERIODIC SOLUTIONS OF SYSTEM (1)

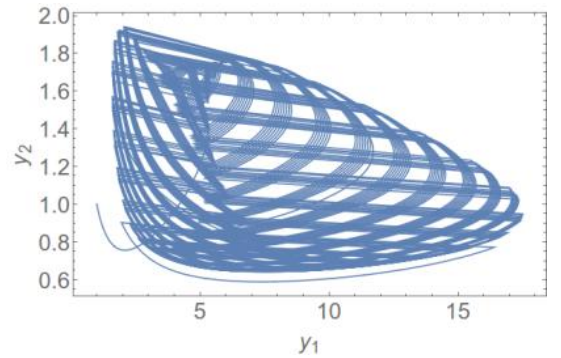

FIGURE IV. PHASE PORTRAIT OF GUI STRANGE ATTRACTOR OF SYSTEM (1)

\section{ACKNOWLEDGMENT}

This work is supported jointly by the Natural Sciences Foundation of China under Grant No. 60963025, Natural Sciences Foundation of Hainan Province under Grant No.613166.

\section{REFERENCES}

[1] D.D. Bainov, and P.S. Simeonov, Systems with Impulse Effect: Stability Theory and Applications, Ellis Horwood Limited, Chichester, 1989.

[2] D.D. Bainov, and P.S. Simeonov, Impulsive Differential Equations: Periodic Solutions and Applications, Longman Scientific \& Technical, New York, 1993.

[3] G.R. Jiang, Q.S. Lu, and L.N. Qian, "Complex dynamics of a Holling type II prey-predator system with state feedback control," Chaos Solitons Fractals, vol. 31, pp:448-461, 2007.

[4] J. Zhang, and Z.J. Gui, "Existence and stability of periodic solutions of high-order Hopfield neural networks with impulses and delays," Journal of Computational and Applied Mathematics, vol. 224, pp. 602-613, 2009

[5] J. Zhang, and Z.J. Gui, "Periodic solutions of nonautonomous cellular neural networks with impulses and delays," Nonlinear Analysis: Real World Applications, vol. 10, pp. 1891-1903, 2009.

[6] C.B. Xing, and Z.J. Gui, "Periodic Solution of Impulsive predator-prey System with Holling-Tanner Type Functional Response," In: L. Chen, et al (eds.). Advances on Biomathematics. Liverpool: World Academic Union (World Academic Press). pp. 58-62, 2008.

[7] J. Zhang, and Z.J. Gui, "Periodic Solution of Predator-Prey Models with the Beddington-DeAngelis Functional Response Governed by Impulsive Differential Equations," In: L. Chen, et al (eds.). Advances on Biomathematics. Liverpool: World Academic Union (World Academic Press). pp. 265-268, 2008.

[8] S.J. Kunwer, and D. Joydip, "Hybrid approach for pest control with impulsive releasing of natural enemies and chemical pesticides: A plantpestnatural enemy model," Nonlinear Analysis: Hybrid System, vol. 12, pp. 79-92, 2014.

[9] W.C Zhao, Y.L. Liu, T.Q. Zhang, and X.Z. Meng, "Geometric analysis of an integrated pest management model including two state impulses," Abstract and Applied Analysis, pp. 1-18, 2014.

[10] R.E. Gaines, J.L. Mawhin. Coincidence degree and nonlinear differential equations. Springer-Verlag, Berlin. 1977. 